\title{
Una foto che racconta un pezzo di storia presente, passata e futura dello studio dei disturbi dell'alimentazione e dell'obesità
}

\author{
Daniele Di Pauli \\ Psicologo, Psicoterapeuta Verona, Italy
}

\section{Parole chiave}

Disturbi dell'alimentazione

Obesità

Terapia cognitivo comportamentale

Trattamento

Prevenzione

Stigma

\begin{abstract}
Riassunto
Scopo dell'articolo è fare una breve sintesi dei principali contributi di Fairburn, Brownell e Dalle Grave nel campo dei disturbi dell'alimentazione e dell'obesità, e le influenze che hanno avuto l'uno sull'altro. Sono descritti i momenti principali che hanno portato dalla prima teorizzazione di un modello di trattamento della bulimia nervosa, fatta da Fairburn nel 1981, allo sviluppo della CBT-E per tutte le categorie diagnostiche dei disturbi dell'alimentazione nei primi anni 2000 (in seguito adattata da Dalle Grave per essere applicata in setting multipli di cura - CBT-E Multistep - e agli adolescenti). Successivamente viene descritto il lavoro iniziale di Brownell sul trattamento e sulla prevenzione dell'obesità seguito dalla CBT-OB di Fairburn e colleghi, rivista e approfondita da Dalle Grave nel corso di questi ultimi anni.
\end{abstract}

\begin{abstract}
Aim of the article is to briefly summarize the main contributions of Fairburn, Brownell and Dalle Grave in the field of eating disorders and obesity, and the influences they had one on the another. The article describes the main moments that led from the first theorization of a treatment model of bulimia nervosa made by Fairburn in 1981 to the development of CBT-E for all diagnostic categories of eating disorders in the early 2000s (later adapted are described by Dalle Grave to be applied in multiple settings of care - CBT-E Multistep - and adolescents). Subsequently, it describes the Brownell's initial work on the treatment and prevention of obesity, followed by the CBT-OB developed by Fairburn and colleagues, reviewed and deepened by Dalle Grave during these last years.
\end{abstract}

Copyright @ 2019 Daniele Di Pauli. This is an open-access article distributed under the terms of the Creative Commons Attribution License (CC BY). The use, distribution or reproduction in other forums is permitted, provided the original author(s) and the copyright owner(s) are credited and that the original publication in this journal is cited, in accordance with accepted academic practice. No use, distribution or reproduction is permitted which does not comply with these terms.

Daniele Di Pauli, PsyD (凶) danieledipauli@hotmail.com

Ricevuto: 30 Maggio 2019; Accettato: 16 Giugno 2019; Pubblicato online: 25 Giugno 2019. doi:10.32044/ijedo.2019.06 
Surfando su internet mi sono imbattuto in una foto: ci sono un italiano, un inglese e un americano... non è l'inizio di una barzelletta, ma ciò che è scolpito in una fotografia che raffigura tre tra le più importanti personalità nel panorama mondiale dello studio e del trattamento dei disturbi dell'alimentazione e dell'obesità. Per chi studia con passione queste tematiche è come per un fan del rock che ha di fronte contemporaneamente i Beatles, i Rolling Stones e i Pink Floyd. Paragoni a parte, Brownell, Fairburn e Dalle Grave sono sinonimi di un trattamento dei disturbi dell'alimentazione e dell'obesità fatto di continua ricerca, innovazione, scientificità e passione.

Scopo dell'articolo è fare una breve sintesi dei principali contributi dei tre ricercatori nel campo dei disturbi dell'alimentazione e dell'obesità, e le influenze che hanno avuto l'uno sull'altro a dimostrare come la scienza non sia una cosa propria ma da condividere.

Christopher Fairburn ${ }^{1}$, classe 1950, psichiatra e ricercatore, professore emerito di psichiatria all'Università di Oxford, è noto per le sue ricerche sullo sviluppo, sulla valutazione $\mathrm{e}$ diffusione di trattamenti psicologici, in particolare per i disturbi dell'alimentazione.

Nel 1981, a due anni dalla prima descrizione della bulimia nervosa fatta da Russell che la definì come "una variante infausta dell'anoressia nervosa” (Russell, 1979), Fairburn pubblica "A Cognitive behavioural approach to the treatment of bulimia" in cui per la prima volta si parla di psicopatologia specifica centrale e di meccanismi di mantenimento, e costruisce una terapia che interviene su questi processi (Fairburn, 1981). In poche parole, non basta normalizzare alcuni aspetti, per esempio il peso o eliminare gli episodi di abbuffata, ma è necessario lavorare sul nucleo psicopatologico centrale del disturbo (cioè l'eccessiva valutazione del peso, della forma del corpo e del controllo dell'alimentazione). Questo lavoro rappresenta un protocollo dettagliato di cura per una patologia decritta come intrattabile, che ha stimolato la diffusione del trattamento cognitivo comportamentale nei servizi pubblici e privati dei paesi occidentali divenendo il primo trattamento psicologico approvato dalle linee guida NICE (National Collaborating Centre for Mental Health, 2004).

Dal 1981 la ricerca di Fairburn e collaboratori non si è fermata fino ad arrivare a sviluppare, nei primi anni 2000, con il gruppo del Centre for Research on Eating Disorders at Oxford (CREDO), la terapia cognitivo comportamentale migliorata (CBT-E) dove "E" sta proprio per Enhanced, cioè migliorata (Fairburn, 2008; Fairburn, Cooper, \& Shafran, 2003). La CBT-E è stata progettata per essere più efficace ri- spetto al trattamento della bulimia nervosa con l'obiettivo di trattare in modo flessibile e individualizzato la psicopatologia specifica del disturbo dell'alimentazione piuttosto che le singole categorie diagnostiche (ipotesi transdiagnostica). Transdiagnostica significa che la teoria (e la terapia derivata da essa) è applicabile, con minime modifiche, a tutte le categorie diagnostiche dei disturbi dell'alimentazione. La CBT-E ha dimostrato di essere efficace nel trattamento di tutte le forme di disturbo dell'alimentazione osservate negli adulti (Byrne et al., 2017; Fairburn et al., 2015; Fairburn et al., 2013; Poulsen et al., 2014) ed è l'unico trattamento raccomandato dalle linee guida NICE per tutte le categorie diagnostiche dei disturbi dell'alimentazione (National Guideline Alliance, 2017).

Un altro merito di Fairburn e del suo gruppo di ricerca è stato lo sviluppo di alcune misure che valutano la psicopatologia del disturbo dell'alimentazione (EDE, EDE-Q) (Fairburn, Cooper, \& O'Connor, 2008a, 2008b) e il suo grado di danno (CIA) (Bohn et al., 2008) che sono utilizzate in tutto il mondo. Queste misure sono state validate in italiano da Simona Calugi e colleghi (Calugi et al., 2017; Calugi, Ricca, et al., 2015; Calugi et al., 2018).

Un'ulteriore, e più recente, evoluzione della CBT-E è nata in Italia grazie al costante ed appassionato lavoro di Riccardo Dalle Grave, classe 1957, laurea in medicina e chirurgia, specializzazione in endocrinologia, in scienze dell'alimentazione e in psicoterapia cognitivo comportamentale. In una proficua collaborazione con Fairburn e il gruppo di ricerca CREDO di Oxford, Dalle Grave, con l'aiuto dei colleghi del gruppo ADA di Verona e dell'Unità di Riabilitazione Nutrizionale di Villa Garda, ha sviluppato la CBT-E multistep (Dalle Grave, 2013; Dalle Grave, 2015) e la CBT-Ea per gli adolescenti (Dalle Grave, 2019a; Dalle Grave, Calugi, \& Sartirana, 2018).

La CBT-E multistep permette di adattare la CBT-E a diversi livelli di cura come il trattamento ambulatoriale, il trattamento ambulatoriale intensivo e il trattamento ospedaliero, mantenendo integre la teoria e le procedure che la caratterizzano. La CBT-E multistep ha permesso di offrire un'alternativa, basata sull'evidenza, a un ampio gruppo di pazienti che non rispondono al trattamento ambulatoriale o le cui condizioni fisiche non ne permettono la gestione in un setting ambulatoriale (Dalle Grave, Calugi, Conti, Doll, \& Fairburn, 2013; Dalle Grave, Calugi, El Ghoch, Conti, \& Fairburn, 2014).

La CBT-Ea ( $\mathrm{a}=$ adolescenti) è nata dall'osservazione che le giovani affette da disturbi dell'alimentazione hanno la stessa psicopatologia specifica delle pazienti adulte. La CBT-Ea

\footnotetext{
${ }^{1}$ Per un approfondimento del lavoro di Fairburn sui DA si consiglia al lettura di "CBT e disturbi dell'alimentazione: Passato, Presente e Futuro. Lettura magistrale del prof. Fairburn a Villa Garda per concludere la sua carriera”.
} 
sostiene che la paziente adolescente possa essere aiutata a riprendere il controllo svolgendo un ruolo attivo e non necessita di un controllo esterno, come sostenuto, per esempio, dal Trattamento Basato sulla Famiglia o FBT (Lock \& Le Grange, 2013). L'attenzione alla motivazione dell'adolescente è continua durante tutto il corso del trattamento. Lo stile della CBTEa è simile ad altre ad altre forme di CBT per quanto riguarda la creazione di un rapporto di collaborazione tra il terapeuta e il paziente, che lavorano assieme, come una squadra, per affrontare i processi che mantengono il disturbo dell'alimentazione (empirismo collaborativo). Tutte le procedure della CBT-Ea sono progettate per far sentire in controllo il paziente adolescente, coinvolgendolo attivamente in tutte le fasi della cura: dalla decisione di iniziare il trattamento, alla scelta dei problemi da affrontare e delle procedure da adottare per superare il suo disturbo dell'alimentazione. Questo approccio è fondamentale perché i pazienti adolescenti con disturbi dell'alimentazione hanno la necessità di sentirsi in controllo in generale (Fairburn, Shafran, \& Cooper, 1999). Al paziente è comunicato che la guarigione dal disturbo dell'alimentazione è difficile, ma ne vale sempre la pena; per tale motivo al paziente è chiesto di considerare il trattamento come una priorità. È anche fondamentale che il terapeuta si assicuri che il paziente capisca sempre quello che sta succedendo nel trattamento e che lo incoraggi a diventare un attivo partecipante nel processo di cambiamento.

La CBT-Ea è stata valutata in quattro studi di coorte che, ottenendo dei risultati promettenti (Calugi, Dalle Grave, Sartirana, \& Fairburn, 2015; Dalle Grave, Calugi, Doll, \& Fairburn, 2013; Dalle Grave et al., 2014; Dalle Grave, Calugi, Sartirana, \& Fairburn, 2015), hanno portato le linee guida NICE a raccomandare questa forma di cura come alternativa al FBT per i giovani affetti da disturbi dell'alimentazione (National Guideline Alliance, 2017).

Kelly Brownell, classe 1951, psicologo clinico noto per il suo lavoro sull'obesità e sulla politica alimentare, è stato fondatore e direttore del Rudd Center for Obesity and Food Policy all'Università di Yale ed attualmente è direttore del World Food Policy Center alla Duke University. Sebbene il suo filone di interesse oggi sia legato alla prevenzione dell'obesità e alla politica alimentare internazionale, in passato si è occupato maggiormente del trattamento dell'obesità.

Brownell è stato allievo di G. Terence Wilson specialista nel trattamento dei disturbi dell'alimentazione e dell'obesità e nei meccanismi psicologici del cambiamento. A fine anni '70 ha lavorato con Albert Stunkard e nel corso di quell'esperienza ha coniato il termine "effetto yo-yo" della dieta (Brownell, Greenwood, Stellar, \& Shrager, 1986; Brownell \& Rodin, 1994). Il suo contributo scientifico, in particolare riguardo alla pre- venzione dell'obesità, ha portato la prestigiosa rivista Time a inserirlo, nel 2006, tra le 100 persone più influenti del pianeta.

Un aspetto centrale del lavoro di Brownell è stato quello di spostare l'attenzione da interventi che coinvolgono il singolo individuo (responsabilità personale) a interventi che agiscano sull'ambiente da lui coniato come "tossico" (Brownell et al., 2010). Il tema della responsabilità personale nella prevenzione e gestione dell'obesità introduce a un filone di ricerca stimolante e fondamentale che ha visto in Brownell un pioniere; quello dello stigma sociale verso l'obesità e le persone che ne sono affette. Nella nostra società le persone glorificano la magrezza e disprezzano l'obesità vista come segno di pigrizia, golosità e non curanza che detronizza l'obesità da malattia cronica, e multifattoriale, a colpa.

Brownell e i suoi colleghi hanno pubblicato diversi lavori sul tema dello stigma tra cui una importante review del 2001 (Puhl \& Brownell, 2001) e un libro "Weight bias: Nature, consequences and remedies" (Brownell, Puhl, Schwartz, \& Rudd, 2005) che sono riferimenti necessari per chi desidera approfondire questa tematica, che nell'ultimo decennio ha avuto un aumento significativo di attenzione e pubblicazioni. Lo stigma ponderale e soprattutto la sua interiorizzazione (che significa che la stessa persona con obesità crede che gli stereotipi negativi siano veri) possono risultare più dannosi delle complicanze mediche dovute al peso e influire negativamente sul trattamento, capacità di perdere e mantenere il peso, minando la salute fisica e psicologica della persona che lo sperimentata. Per questi motivi la gestione e prevenzione dell'obesità non possono essere fatte senza una riduzione dello stigma sociale che può rappresentare una vera e propria barriera al trattamento e all'attenzione dell'opinione pubblica e del mondo politico (Di Pauli, 2016).

Brownell ha inoltre contribuito in modo fondamentale allo sviluppo del trattamento comportamentale dell'obesità con il libro "The LEARN program for weight management" acronimo di lifestyle, exercise, attitudes, relationships and nutrition (Brownell, 2000). Lo scopo del libro, suddiviso in 16 lezioni settimanali, è quello di aiutare le persone a sviluppare un atteggiamento "flessibile" e le abilità necessarie per perdere e mantenere il peso nel tempo. Nel testo si trovano quei principi fondamentali (per es. monitoraggio alimentare, ruolo dei pensieri disfunzionali nella perdita di peso, gestione delle ricadute) che ritroviamo nella terapia cognitivo comportamentale dell'obesità descritta da Cooper e colleghi nel libro "Cognitive behavioral Treatment of Obesity: A clinician guide" (Cooper, Fairburn, \& Hawker, 2003). La terapia che viene descritta segue l'importante articolo del 2001 in cui gli autori hanno cercato di analizzare i fattori che portano, la quasi totalità delle persone che perdono peso o desiderano perdere peso, al recupero 
del peso perduto o all'abbandono del tentativo di perdere peso (Cooper \& Fairburn, 2001). Una progressiva diminuzione della credenza del paziente di controllare il proprio peso (che in genere arriva dopo 4-6 mesi quando la perdita di peso raggiunge il suo plateau) e la conseguente perdita di motivazione dovuta all'idea di non raggiungere quegli obiettivi che si pensava di raggiungere con la perdita di peso (che gli autori hanno chiamato "obiettivi primari") sono i fattori implicati nella difficoltà a mantenere il peso perduto o che portano a un abbandono del programma. Carattere distintivo della CBT dell'obesità infatti è avere due fasi distinte del trattamento: una che si focalizza sulla perdita di peso e una sul mantenimento, e accettazione, del peso perduto e risultati raggiunti.

Dalle Grave nei testi "Terapia cognitivo comportamentale dell'obesità" (Dalle Grave, 2001), "Perdere e mantenere il peso" (Dalle Grave, 2004, 2019b) e nel recente "Treating obesity with personalized cognitive behavioral therapy" (Dalle Grave, Sartirana, El Ghoch, \& Calugi, 2018) ha ripreso la teorizzazione di Fairburn e colleghi portando i 9 moduli originali a 6 , con particolare enfasi sull'accettazione dei risultati raggiunti durante la fase 1 , affrontando l'eventuale insoddisfazione nei confronti della perdita di peso, che permettono un lavoro più efficace nella fase 2. Nell'ultimo libro Dalle Grave sottolinea anche l'importanza di un trattamento individualizzato che è come un abito cucito su misura da un sarto in base ai bisogni e caratteristiche della persona che chiede aiuto. Infatti, quando si parla di obesità bisogna usare il plurale (in inglese obesities) che significa che le obesità sono diverse tra loro e non possono essere trattate tutte alla stessa maniera (anche questo un limite degli interventi che si basano sul paradigma della responsabilità personale). Il trattamento va costruito sul paziente adattando ai suoi bisogni e caratteristiche le tecniche e strategie tipiche della CBT.

$\mathrm{Ci}$ vorrebbero pagine e pagine per rendere giustizia al lavoro di questi tre professionisti e dell'importanza storica, scientifica ed emotiva che ha la foto che fa da musa a questo articolo. Gli Stadio cantavano "chiedi chi erano i Beatles" e fin che scrivevo avevo il ritornello di questa famosa canzone in mente.

Sicuramente se qualcuno dovesse chiedere, a chi lavora nel campo dei disturbi dell'alimentazione e dell'obesità, "Ma chi sono Brownell, Fairburn e Dalle Grave?" ci sarebbe una bella storia da raccontare, anzi tre, che si incontrano, si intersecano e trovano numerosi punti in comune. Una sorta di storia "transdiagnostica" su cui speriamo ci sia ancora molto da scrivere, e imparare, e magari qualcuno che ora sta leggendo, un domani, potrà aggiungersi come quarta persona nella foto.

Del resto, i Beatles erano in 4.

Ma chi erano mai questi Beatles?

\section{Bibliografia}

Bohn, K., Doll, H. A., Cooper, Z., O'Connor, M., Palmer, R. L., \& Fairburn, C. G. (2008). The measurement of impairment due to eating disorder psychopathology. Behaviour Research and Therapy, 46(10), 1105-1110. doi:10.1016/j.brat.2008.06.012

Brownell, K. D. (2000). The LEARN program for weight management 2000: Lifestyle, exercise, attitudes, relationships, nutrition. Dallas, Tex: American Health Pub. Co.

Brownell, K. D., Greenwood, M. R., Stellar, E., \& Shrager, E. E. (1986). The effects of repeated cycles of weight loss and regain in rats. Physiology and Behavior, 38(4), 459-464.

Brownell, K. D., Kersh, R., Ludwig, D. S., Post, R. C., Puhl, R. M., Schwartz, M. B., \& Willett, W. C. (2010). Personal responsibility and obesity: a constructive approach to a controversial issue. Health Affairs, 29(3), 379-387. doi:10.1377/hlthaff.2009.0739

Brownell, K. D., Puhl, R. M., Schwartz, M. B., \& Rudd, L. (2005). Weight bias: Nature, consequences, and remedies. New York: Guilford Press.

Brownell, K. D., \& Rodin, J. (1994). Medical, metabolic, and psychological effects of weight cycling. Archives of Internal Medicine, 154(12), 1325-1330.

Byrne, S., Wade, T., Hay, P., Touyz, S., Fairburn, C. G., Treasure, J., . . Crosby, R. D. (2017). A randomised controlled trial of three psychological treatments for anorexia nervosa. Psychological Medicine, 1-11. doi:10.1017/s0033291717001349

Calugi, S., Dalle Grave, R., Sartirana, M., \& Fairburn, C. G. (2015). Time to restore body weight in adults and adolescents receiving cognitive behaviour therapy for anorexia nervosa. Journal of Eating Disorders, 3, 21. doi:10.1186/s40337-015-0057-z

Calugi, S., Milanese, C., Sartirana, M., El Ghoch, M., Sartori, F., Geccherle, E., . . Dalle Grave, R. (2017). The Eating Disorder Examination Questionnaire: reliability and validity of the Italian version. Eating and Weight Disorders, 22(3), 509-514. doi:10.1007/s40519-016-0276-6

Calugi, S., Ricca, V., Castellini, G., Lo Sauro, C., Ruocco, A., Chignola, E., . . Dalle Grave, R. (2015). The Eating Disorder Examination: Reliability and validity of the Italian version. Eating and Weight Disorders, 20(4), 505511. doi:10.1007/s40519-015-0191-2

Calugi, S., Sartirana, M., Milanese, C., El Ghoch, M., Riolfi, F., \& Dalle Grave, R. (2018). The clinical impairment assessment questionnaire: validation in Italian patients with eating disorders. Eating and Weight Disorders, 23(5), 685-694. doi:10.1007/s40519-018-0477-2 
Cooper, Z., \& Fairburn, C. G. (2001). A new cognitive behavioural approach to the treatment of obesity. Behaviour Research and Therapy, 39(5), 499-511.

Cooper, Z., Fairburn, C. G., \& Hawker, D. M. (2003). Cognitive-behavioral treatment of obesity: a clinician's guide. New York: Guilford Press.

Dalle Grave, R. (2001). Terapia Cognitivo Comportamentale dell'obesità. Verona: Positive Press:.

Dalle Grave, R. (2004). Perdere e mantenere il peso. Un nuovo programma cognitivo comportamentale. Verona: Positive Press.

Dalle Grave, R. (2013). Multistep cognitive behavioral therapy for eating disorders: Theory, practice, and clinical cases. New York: Jason Aronson.

Dalle Grave, R. (2015). La terapia cognitivo comportamentale multistep dei disturbi dell'alimentazione. Teoria, trattamento e casi clinici. Firenze: Eclipsi.

Dalle Grave, R. (2019a). Cognitive-behavioral therapy in adolescent eating disorders. In J. Hebebrand \& B. HerpertzDahlmann (Eds.), Eating disorders and obesity in children and adolescents (pp. 111-116). Philadelphia: Elsevier.

Dalle Grave, R. (2019b). Perdere e mantenere il peso. Un programma di modificazione dello stile di vita basato sulla terapia cognitivo comportamentale. Verona: Positive Press.

Dalle Grave, R., Calugi, S., Conti, M., Doll, H., \& Fairburn, C. G. (2013). Inpatient cognitive behaviour therapy for anorexia nervosa: a randomized controlled trial. Psychotherapy and Psychosomatics, 82(6), 390-398. doi:10.1159/000350058

Dalle Grave, R., Calugi, S., Doll, H. A., \& Fairburn, C. G. (2013). Enhanced cognitive behaviour therapy for adolescents with anorexia nervosa: an alternative to family therapy? Behaviour Research and Therapy, 51(1), R9-R12. doi:10.1016/j.brat.2012.09.008

Dalle Grave, R., Calugi, S., El Ghoch, M., Conti, M., \& Fairburn, C. G. (2014). Inpatient cognitive behavior therapy for adolescents with anorexia nervosa: immediate and longer-term effects. Frontiers in Psychiatry, 5, 14. doi:10.3389/fpsyt.2014.00014

Dalle Grave, R., Calugi, S., \& Sartirana, M. (2018). Manuale di terapia cognitivo comportamentale dei disturbi dell'alimentazione nell'adolescenza (CBT-Ea). Dal trattamento ambulatoriale al ricovero riabilitativo. Verona: Positive Press.

Dalle Grave, R., Calugi, S., Sartirana, M., \& Fairburn, C. G. (2015). Transdiagnostic cognitive behaviour therapy for adolescents with an eating disorder who are not underweight. Behaviour Research and Therapy, 73, 79-82. doi:10.1016/j.brat.2015.07.014
Dalle Grave, R., Sartirana, M., El Ghoch, M., \& Calugi, S. (2018). Treating Obesity with Personalized Cognitive Behavioral Therapy. Cham: Springer.

Di Pauli, D. (2016). Where's Icarus? Stigma based on weight that no one wants to see. A candid answer to "A candid discussion of obesity". Eating and Weight Disorders, 21(3), 349-351. doi:10.1007/s40519-016-0294-4

Fairburn, C. (1981). A cognitive behavioural approach to the treatment of bulimia. Psychological Medicine, 11(4), 707711. doi:10.1017/S0033291700041209

Fairburn, C. G. (2008). Cognitive behavior therapy and eating disorders. New York: Guilford Press.

Fairburn, C. G., Bailey-Straebler, S., Basden, S., Doll, H. A., Jones, R., Murphy, R., . . Cooper, Z. (2015). A transdiagnostic comparison of enhanced cognitive behaviour therapy (CBT-E) and interpersonal psychotherapy in the treatment of eating disorders. Behaviour Research and Therapy, 70, 64-71. doi:10.1016/j.brat.2015.04.010

Fairburn, C. G., Cooper, Z., Doll, H. A., O’Connor, M. E., Palmer, R. L., \& Dalle Grave, R. (2013). Enhanced cognitive behaviour therapy for adults with anorexia nervosa: a UK-Italy study. Behaviour Research and Therapy, 51(1), R2-8. doi:10.1016/j.brat.2012.09.010

Fairburn, C. G., Cooper, Z., \& O’Connor, M. (2008a). Eating disorder examination In C. G. Fairburn (Ed.), Cognitive behavior therapy and eating disorders (pp. 265-308). Guilford Press: New York.

Fairburn, C. G., Cooper, Z., \& O’Connor, M. (2008b). Eating Disorder Examination Questionnaire (EDE-Q 6.0). In C. G. Fairburn (Ed.), Cognitive behavior therapy and eating disorders (pp. 309-313). Guilford Press: New York.

Fairburn, C. G., Cooper, Z., \& Shafran, R. (2003). Cognitive behaviour therapy for eating disorders: a "transdiagnostic" theory and treatment. Behaviour Research and Therapy, 41(5), 509-528.

Fairburn, C. G., Shafran, R., \& Cooper, Z. (1999). A cognitive behavioural theory of anorexia nervosa. Behaviour Research and Therapy, 37(1), 1-13.

Lock, J., \& Le Grange, D. (2013). Treatment manual for anorexia nervosa: A family-based approach (2nd ed.). New York: Guilford Press.

National Collaborating Centre for Mental Health. (2004). National Institute for Health and Clinical Excellence: Guidance. In Eating Disorders: Core interventions in the treatment and management of anorexia nervosa, bulimia nervosa and related eating disorders. Leicester (UK): British Psychological Society (UK).

National Guideline Alliance. (2017). National Institute for Health and Care Excellence: Clinical Guidelines. In 
Eating disorders: Recognition and treatment. London: National Institute for Health and Care Excellence (UK).

Poulsen, S., Lunn, S., Daniel, S. I., Folke, S., Mathiesen, B. B., Katznelson, H., \& Fairburn, C. G. (2014). A randomized controlled trial of psychoanalytic psychotherapy or cognitive-behavioral therapy for bulimia nervosa. American Journal of Psychiatry, 171(1), 109-116. doi:10.1176/appi.ajp.2013.12121511
Puhl, R., \& Brownell, K. D. (2001). Bias, discrimination, and obesity. Obesity Research, 9(12), 788-805. doi:10.1038/ oby. 2001.108

Russell, G. (1979). Bulimia nervosa: an ominous variant of anorexia nervosa. Psychological Medicine, 9(3), 429-448. 